\title{
Field Theoretical Construction of an Infinite Set of Quantum Commuting Operators Related with Soliton Equations
}

\author{
Ryu Sasaki and Itaru Yamanaka \\ Research Institute for Theoretical Physics, Hiroshima University, Takehara, \\ Hiroshima, 725 Japan
}

\begin{abstract}
The quantum version of an infinite set of polynomial conserved quantities of a class of soliton equations is discussed from the point of view of naive continuum field theory. By using techniques of two dimensional field theories, we show that an infinite set of quantum commuting operators can be constructed explicitly from the knowledge of its classical counterparts. The quantum operators are so constructed as to coincide with the classical ones in the $\hbar \rightarrow 0$ limit ( $\hbar$; Planck's constant divided by $2 \pi$ ). It is expected that the explicit forms of these operators would shed some light on the structure of the infinite dimensional Lie algebras which underlie a certain class of quantum integrable systems.
\end{abstract}

\section{Introduction}

The existence of an infinite set of polynomial conserved quantities in involution to each other under a certain Poisson bracket is one of the most characteristic features of classical soliton theories [1] in $1+1$ dimensions, which are the best understood nonlinear field theories. The infinite set of conserved quantities is the cornerstone for the complete integrability of classical systems. The situation is the same for quantum theories. Therefore a natural question arises whether the classical infinite dimensional symmetry survives quantization. Namely, do we get an infinite set of quantum commuting operators which reduces to the classical one in the limit of $\hbar \rightarrow 0$ ( $\hbar$; the Planck constant divided by $2 \pi$ ), or do we not, due to some anomalies caused by the high nonlinearity of the interaction? Usually this problem is investigated system by system in terms of the quantum inverse method [2] or by the transfer matrix approach based on a lattice [3]. In such approaches, however, the field theoretical aspects are made rather obscure behind a strong algebraic structure like the Yang-Baxter algebra.

In this connection we should mention one of the successful examples of the quantum inverse method, the nonlinear Schrödinger equation,

$$
i \psi_{t}+\psi_{x x}-\kappa \psi^{\dagger} \psi \psi=0 \text {. }
$$


In this case an infinite set of quantum commuting operators (the scattering data) in terms of the original field operators in the continuum is explicitly constructed [4] and its relationship with the Bethe ansatz is clarified. (However, as for the quantum version of the polynomial conserved quantities, only a few lowest members have been identified. It is by no means obvious whether the higher members do exist or not.) This success is due to the exceptional situation of the nonlinear Schrödinger equation that the creation operators and the annihilation operators are clearly separated at the field operator level, i.e., $\psi$ for annihilation and $\psi^{\dagger}$ for creation, thereby the direct generalization of the classical linear scattering method to the quantum case is justified. In the other soliton theories, for example, the Korteweg de Vries (KdV) equation, the Modified KdV (MKdV) equation or the sine-Gordon equation, both creation and annihilation parts are contained in one hermitian field operator. So far, at least to our knowledge, the direct application of the quantum inverse method to derive the explicit forms of quantum commuting operators for these equations is not successful.

In this paper we will investigate the above question, namely existence and explicit construction of an infinite set of quantum commuting operators for soliton equations like the $\mathrm{KdV}$ eq and the $\mathrm{MKdV}$ eq, from the quantum field theoretical point of view, as an alternative approach to the existing formulations. Use is made of the tools of conventional field theories, for example, propagators, Feynman diagrams and Wick's theorem, etc. Our discussion is mainly based on a class of soliton equations represented by the MKdV equation, Eq. (2.1), which is also related with the sine $(h)$-Gordon equation. The situation is almost the same for the $\mathrm{KdV}$ equation case, on which we briefly comment in Sect. 5 . The starting point is that the Poisson bracket characteristic for these classes of soliton equations $(\mathrm{MKdV}, \mathrm{KdV})$ corresponds exactly to the commutation relation of the two dimensional boson fields. Therefore naive quantization in the continuum is straightforward. As for the calculation of commutators among various operators, the techniques of two dimensional field theories are quite useful.

An algorithm to construct the infinite set of quantum commuting operators is obtained and the first six members are calculated explicitly. A preliminary and partial result on these materials has been published in [5]. We believe these explicit examples will provide another interesting path for understanding the nature of quantum infinite dimensional symmetries.

This paper is organized as follows. In Sect. 2 we review briefly the classical theory of the infinite set of conserved quantities for the MKdV eq. In Sect. 3 the quantization procedure and the calculation techniques for commutators based on field theoretical tools are explained. In Sect. 4 an algorithm of constructing the infinite set of quantum commuting operators is derived together with its explicit forms for lower members. In Sect. 5 we briefly discuss the parallel results for the $\mathrm{KdV}$ equation. The final section is devoted to summary and comments.

\section{Classical Theory of an Infinite Set of Polynomial Conserved Quantities Associated with the MKdV Eq}

In order to introduce notation and to be self-contained, we summarize some of the known results of the infinite set of polynomial conserved quantities associated with 
the Modified Korteweg de Vries equation (the MKdV eq)

$$
u_{t}=u_{\sigma \sigma \sigma}-6 u^{2} u_{\sigma} .
$$

Here $u=u(t, \sigma)$ is a real (hermitian) field in $1+1$ dimensions depending on time $(t)$ and space $(\sigma)$ variables and $u_{t}=\partial_{t} u, u_{\sigma}=\partial_{\sigma} u, u_{\sigma \sigma}=\partial_{\sigma}^{2} u, \ldots$, etc. It is assumed that $u$ has continuous $\sigma$-derivatives of all orders. Throughout this paper we impose the periodic boundary condition with a period $2 \pi$,

$$
u(t, \sigma+2 \pi)=u(t, \sigma), \quad 0 \leqq \sigma \leqq 2 \pi .
$$

The reason for this will become clear in later sections. It should be remarked, however, that the explicit forms of conserved densities to be discussed below are the same for the periodic boundary condition case and for the ordinary boundary condition case $[u(\sigma) \rightarrow 0$, as $|\sigma| \rightarrow \infty,-\infty<\sigma<\infty]$.

An infinite set of polynomial conserved quantities $[1,6]$ is given as follows:

$$
I_{n}=-\frac{1}{2 \pi} \int_{0}^{2 \pi} d \sigma \frac{1}{2} u(\sigma) Y_{2 n-1}(u), \quad n=1,2,3, \ldots,
$$

in which $Y_{n}(u)$ is a polynomial in $u$ and its space derivatives $u_{\sigma}, u_{\sigma \sigma}, \ldots$, defined recursively

$$
Y_{n+1}=\partial_{\sigma} Y_{n}+u \sum_{k=1}^{n-1} Y_{k} Y_{n-k}, \quad Y_{1}=-u .
$$

It should be remarked that in Eqs. (2.3), (2.4) and hereafter the time $t$ is always fixed and suppressed. It is easy to show that $u Y_{2 n}$ is a total $\sigma$-derivative giving rise to a trivial conserved quantity. We define the order and the weight of a monomial in $u$, $u_{\sigma}, u_{\sigma \sigma}, \ldots$,

as follows ${ }^{1}$

$$
M=u^{n_{0}} u_{\sigma}^{n_{1}} \ldots u_{m \sigma}^{n_{m}}
$$

$$
\operatorname{order}(M)=\sum n_{k}, \quad \text { weight }(M)=\sum(k+1) n_{k} .
$$

Then we find that the polynomial $u Y_{n}$ has a uniform weight

$$
\text { weight }\left(u Y_{n}\right)=n+1 \text {, }
$$

but its component monomials have different orders, all even,

$$
2 \leqq \text { order of each term in } u Y_{2 n-1}, u Y_{2 n} \leqq 2 n \text {. }
$$

To sum up, the conserved quantity $I_{n}$ has even order terms only with a uniform weight $2 n$.

A remarkable and highly nontrivial result of the soliton theory is that the infinite set of conserved quantities, $I_{n}, n=1,2,3, \ldots$, are in involution to each other with respect to the following Poisson bracket [8-11]

$$
\begin{aligned}
& \left\{u(\sigma), u\left(\sigma^{\prime}\right)\right\}=2 \pi \partial_{\sigma} \delta\left(\sigma-\sigma^{\prime}\right), \\
& \left\{I_{n}, I_{m}\right\}=0, n, m=1,2, \ldots, .
\end{aligned}
$$

\footnotetext{
${ }^{1}$ Our notation and definition slightly differ from those given for the $\mathrm{KdV}$ eq in [7]
} 
By adopting $I_{2}$ [for its explicit form, see Eq. (4.2)] as a Hamiltonian, we get the MKdV eq (2.1) as a canonical equation

$$
\partial_{t} u(\sigma)=\left\{u(\sigma), I_{2}\right\} .
$$

From the above involution property we find that the set $\left\{I_{n}\right\}$ is conserved for a large class of evolution equations

$$
\partial_{t} u(\sigma)=\{u(\sigma), H\},
$$

in which the Hamiltonian $H$ is given by

$$
H=\sum_{n} h_{n}(t) I_{n},
$$

with arbitrary time dependent functions $h_{n}(t), n=1,2, \ldots$, . In fact we have

$$
\partial_{t} I_{m}=\left\{I_{m}, H\right\}=\sum_{n} h_{n}(t)\left\{I_{m}, I_{n}\right\}=0 .
$$

Thus we see that the infinite set of conserved quantities together with the Poisson bracket characterize the class of soliton equations. Let us call this class of soliton equations, Eq. (2.11) the MKdV hierarchy.

A few remarks about the Poisson bracket are in order. Let us denote by $P$ the integral of a local polynomial $p$ in $u, u_{\sigma}, u_{\sigma \sigma}, \ldots$,

$$
P=\frac{1}{2 \pi} \int_{0}^{2 \pi} d \sigma p\left(u, u_{\sigma}, u_{\sigma \sigma}, \ldots\right),
$$

and by $V$ the linear space spanned by them ${ }^{2}$

$$
V=\{P \mid P \text { given in Eq. (2.14) }\} \text {. }
$$

$V$ is infinite dimensional but its linear subspaces of uniform weight have finite dimensions. If $P$ is of uniform weight, so is $\left\{I_{n}, P\right\}$

$$
\text { weight }\left\{I_{n}, P\right\}=\text { weight }(P)+2 n-1 \text {. }
$$

In particular, the Poisson bracket with $I_{2},\left\{I_{2}, P\right\}$, increases the weight by 3 and the order by 0 and 2 . About the uniqueness of the polynomial conserved quantities for the MKdV hierarchy, we have the following.

Theorem 1. If $\left\{I_{2}, P\right\}=0$, then

$$
P=\sum_{n=0} c_{n} I_{n}
$$

in which $I_{0}=\int_{0}^{2 \pi} u d \sigma$, and $c_{n}$ are constants. In particular, if $P$ is of even order and odd weight and $\left\{I_{2}, P\right\}=0$, then $P=0$.

The proof is obtained by a slight modification of the corresponding theorem for the $\mathrm{KdV}$ equation given in [7].

Corollary. For given $Q \in V$, a linear equation for $P \in V$,

$$
\left\{I_{2}, P\right\}=Q,
$$

${ }^{2}$ For details of this linear space, see [7] 
has a unique solution modulo $I_{n}, n=0,1,2,3, \ldots$, if and only if $Q$ is in the image of a linear operator $\mathscr{M}$

$$
\mathscr{M} ; * \rightarrow\left\{I_{2}, *\right\} \text {. }
$$

The fact that the map $\mathscr{M}$ is not surjective can be easily seen by considering a uniform weight, $w$, slice. As remarked above $\mathscr{M}$ increases the weight by 3 and the dimension of the linear subspace of $V$ with uniform weight $w+3$ is in general higher than that of the linear subspace with weight $w$.

\section{Quantization}

In this section we discuss the quantization of the class of non-linear Hamiltonian dynamics, the MKdV hierarchy, introduced in the preceding section. Here we follow the naive canonical quantization procedure in continuum theory rather than applying the quantum inverse method or the transfer matrix approach. The canonical quantization is achieved by replacing the canonical Poisson bracket by the commutation relation

$$
\left\{q_{r}, p_{s}\right\}=\delta_{r, s} \rightarrow\left[q_{r}, p_{s}\right]=i \hbar \delta_{r, s} .
$$

Since we are interested in the relation between the classical and the quantum soliton theories all the $\hbar$ dependence are displayed explicitly. In the present case the fundamental commutation relation derived from the Poisson bracket (2.8) reads

$$
\left[u(\sigma), u\left(\sigma^{\prime}\right)\right]=i \hbar 2 \pi \partial_{\sigma} \delta\left(\sigma-\sigma^{\prime}\right) .
$$

As is well known in two dimensional field theories as well as in string theories, the above commutation relation is realized by (the derivative of) a hermitian boson field;

$$
\begin{array}{cc}
u(\sigma)=\sum_{n=-\infty}^{\infty} \alpha_{n} e^{-i n \sigma}, & 0 \leqq \sigma \leqq 2 \pi, \\
{\left[\alpha_{m}, \alpha_{n}\right]=\hbar m \delta_{m+n, 0},} & \left(\alpha_{m}\right)^{\dagger}=\alpha_{-m} .
\end{array}
$$

We interpret $\alpha_{m}, m>0(m<0)$ as (annihilation) creation operators and $\alpha_{0}$ as a zero mode. The vacuum $|0\rangle$ is defined by

$$
\alpha_{m}|0\rangle=0, \quad m \geqq 0,
$$

and the Fock space is built by repeated application of creation operators on the state $|0 ; p\rangle$, which is defined by

$$
\alpha_{m}|0: p\rangle=0, \quad m \geqq 1, \quad \alpha_{0}|0 ; p\rangle=p|0 ; p\rangle, \quad p \in \mathbb{R} .
$$

In order to define the quantum version of the non-linear dynamics, Eqs. (2.11), (2.12), we have to specify the operator ordering for $I_{n}, n=1,2,3, \ldots$, . Containing products of operators $u, u_{\sigma}, u_{\sigma \sigma}, \ldots$, at the same space-time point, $I_{n}$ in quantum theory is full of divergences. The simplest way to get rid of the divergence is to adopt the normal product, which we denote by dots::. So we denote by $\hat{I}_{n}$ the quantum operator corresponding to the classical quantity $I_{n}$. It has the following general structure

$$
\hat{I}_{n}=: I_{n}:+\sum_{k=1} \hbar^{k}: I_{n}^{(k)}:, \quad n=1,2,3, \ldots
$$


in which $I_{n}^{(k)}$ are yet unspecified but they should have the form

$$
I_{n}^{(k)}=\frac{1}{2 \pi} \int_{0}^{2 \pi} d \sigma \mathscr{I}_{n}^{(k)}
$$

and $\mathscr{I}_{n}^{(k)}$ is a local ${ }^{3}$ polynomial in $u, u_{\sigma}, u_{\sigma \sigma}, \ldots$, . Namely, $I_{n}^{(k)} \in V$. The classical theory comes in as the $\hbar$-independent part, namely the first term on the r.h.s. of Eq. (3.5).

We are now in a position to pose the question raised in the Introduction in a more definite way.

Question: Is it possible to define $I_{n}^{(k)}$ such that all $\hat{I}_{n}$ commute with each other ${ }^{4}$ ?

$$
\left[\hat{I}_{n}, \hat{I}_{m}\right]=0, \quad n, m=1,2, \ldots
$$

Since $\hbar$ is treated as a free parameter, Eq. (3.7) demands that the coefficients of each power of $\hbar$ should vanish. We impose the following conditions on possible local counterterms $\mathscr{I}_{n}^{(k)}$;

$$
\begin{gathered}
\text { max order }\left(\mathscr{I}_{n}^{(k)}\right)=2(n-k), \\
\text { implying } \mathscr{I}_{n}^{(k)}=0 \quad \text { for } \quad k \geqq n, \\
\text { max weight }\left(\mathscr{I}_{n}^{(k)}\right)=2 n, \\
\mathscr{I}_{n}^{(k)} \text { has even order, even weight terms only } .
\end{gathered}
$$

(ii) Irreducibility,

$$
I_{n}^{(k)} \text { should not contain any of } I_{m} \text { as a part. }
$$

By considering the concrete forms of $I_{n}$, Eq. (2.3), and the condition (3.8e) this can be restated as

$$
\mathscr{I}_{n}^{(k)} \text { should not contain terms of the form } u^{2 m} \text {. }
$$

The necessity of the irreducibility condition is obvious. The meaning of the condition $(3.8 \mathrm{a} \sim \mathrm{c})$ is that the order and the weight of the added terms should not exceed those of the original terms in $I_{n}$. Here one should take into account the fact that $\hbar$ has the order 2 due to the commutation relation (3.1). If one relaxes these conditions then the whole program Eqs. (3.5), (3.7) would be uncontrollable.

In order to facilitate the calculation of commutators of normal ordered polynomials in field operators, we adopt the following technique which is rather common in string theories and in conformally invariant two dimensional field theories. Let us introduce a fictitious "time" variable $\tau$ and define a complex coordinate $z$ by

$$
z=e^{\tau+i \sigma}
$$

\footnotetext{
${ }^{3}$ The locality is essential in order that the resulting integrable quantum field theory defined by $\hat{I}_{n}$ be local

${ }^{4}$ For systems with finite degrees of freedom, similar approach to ours, namely deriving a quantum completely integrable system directly from a classical integrable one by adding appropriate counterterms of order $\hbar$ and higher, has been discussed for several models [12]
} 
and consider the field theory defined on the complex plane

$$
u(z)=\sum_{n} \alpha_{n} z^{-n},
$$

instead of the original theory defined on a circle $S^{1}$. It is obvious that by restricting the coordinate $z$ to a unit circle we regain the original theory. For an arbitrary local polynomial $p$ in the field variables $u, u_{\sigma}, u_{\sigma \sigma}, \ldots$, we have

$$
\frac{1}{2 \pi} \int_{0}^{2 \pi} d \sigma p\left(u, u_{\sigma}, u_{\sigma \sigma}, \ldots,\right)=\frac{1}{2 \pi i} \oint_{0} \frac{d z}{z} p\left(u, D u, D^{2} u, \ldots,\right),
$$

in which

$$
D=i z \partial_{z} .
$$

The integration contour on the r.h.s. of Eq. (3.11) should encircle the origin once and otherwise arbitrary due to the analyticity. Next we define the radial ordering [13] $R$ of two local operators $a(\zeta), b(z)$ with complex coordinates $\zeta$ and $z$,

$$
R a(\zeta) b(z)=\begin{array}{rll}
a(\zeta) b(z), & \text { if } & |\zeta|>|z|, \\
\pm b(z) a(\zeta), & \text { if } & |\zeta|<|z| .
\end{array}
$$

The minus sign is for fermionic operators $a$ and $b$, which we do not consider in the present paper. The propagator of the $u$ field is defined as the vacuum expectation value of the radial ordered product $R u(\zeta) u(z)$,

$$
\hbar \Delta(\zeta, z)=\langle 0|R u(\zeta) u(z)| 0\rangle=\hbar \frac{\zeta z}{(\zeta-z)^{2}} .
$$

Let us denote by $: P$ : the integral of a normal ordered polynomial $p$ as is given in Eq. (3.11),

$$
: P:=\frac{1}{2 \pi i} \oint_{0} \frac{d z}{z}: p(z): .
$$

Similarly $: Q:$ is defined by

$$
: Q:=\frac{1}{2 \pi i} \oint_{0} \frac{d z}{z}: q(z): .
$$

Then by using the definition of the radial ordering and a simple deformation of integral contours, we get the following general formula for the commutator $[: P:$, $: Q:]$, (Fig. 1)

$$
[: P:,: Q:]=\frac{1}{(2 \pi i)^{2}} \oint_{0} \frac{d z}{z} \oint_{c_{z}} \frac{d \zeta}{\zeta} R: p(\zeta):: q(z):
$$

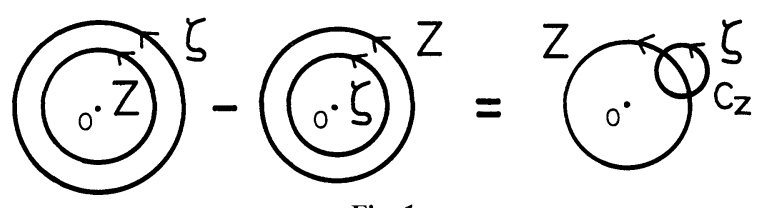

Fig. 1 
in which $c_{z}$ is a small contour encircling the point $z$. By Wick's theorem [14] we expand the radial ordered product $R: p(\zeta):: q(z):$ in terms of the propagator $\Delta(\zeta, z)$. The $k$-times contraction term contains a factor $\hbar^{k} \prod_{i}^{k}\left(D^{n_{i}} \Delta\right)$ and it corresponds to a $(k-1)$ loop Feynman diagram. The $\zeta$-integration is the standard residue calculus. Therefore the resulting commutator, i.e., the r.h.s. of Eq. (3.16) has the same structure as $P$ and $Q$,

$$
[: P:,: Q:]=\frac{1}{2 \pi i} \oint_{0} \frac{d z}{z}: r(z):
$$

in which $r$ is an appropriate polynomial in $u, u_{\sigma}, u_{\sigma \sigma}, \ldots$, .

Let us denote by $\hbar^{k}[: P:,: Q:]_{(k)}$ the contribution from the $k$-times contraction terms for the commutator (3.16). Then obviously we have

$$
[: P:,: Q:]=\sum_{k=1} \hbar^{k}[: P:,: Q:]_{(k)} .
$$

The first contraction term, $k=1$, corresponding to tree diagrams and proportional to $\hbar$, gives the classical contribution as is well known.

$$
[: P:,: Q:]_{(1)}=i:\{P, Q\}: \text {. }
$$

Since the number of contractions cannot exceed either of the maximal order of $p$ and $q$, we have

$$
[: P:,: Q:]_{(l)}=0, \quad \text { if } \quad l \geqq \min (\bar{p}, \bar{q}),
$$

in which $\bar{p}(\bar{q})$ is the maximal order of $p(q)$. It is easy to see that by making a commutator the parity of the order is conserved whereas the parity of the weight is changed. In particular, if $P$ and $Q$ are both of even order and even weight, then $[: P:,: Q:]$ is of even order and odd weight.

\section{Infinite Set of Quantum Commuting Operators}

In this section we give evidence that the infinite set of quantum commuting operators $\hat{I}_{n}$,

$$
\left[\hat{I}_{n}, \hat{I}_{m}\right]=0, \quad n, m=1,2, \ldots,
$$

does exist and that its members can be determined explicitly. As stated in the previous section we look for $\hat{I}_{n}$ in the following form:

$$
\hat{I}_{n}=: I_{n}:+\sum \hbar^{k}: I_{n}^{(k)}:,
$$

in which $I_{n}^{(k)}$ 's $(k \geqq 1)$ satisfying the conditions (3.8) are to be determined. Let us write down possible forms for some lower members explicitly in the notation of Eq. (3.6);

$$
\begin{array}{ll}
\hat{I}_{1}=: I_{1}:, & \mathscr{I}_{1}=\frac{1}{2} u^{2}, \\
\hat{I}_{2}=: I_{2}:, & \mathscr{I}_{2}=\frac{1}{2}\left(-(D u)^{2}-u^{4}\right), \\
\hat{I}_{3}=: I_{3}:+\hbar: I_{3}^{(1)}:+\hbar^{2}: I_{3}^{(2)}:, & \mathscr{I}_{3}=\frac{1}{2}\left(D^{2} u\right)^{2}+5 u^{2}(D u)^{2}+u^{6},
\end{array}
$$




$$
\begin{aligned}
\mathscr{I}_{3}^{(1)}= & a_{1}\left(D^{2} u\right)^{2}+a_{2} u^{2}(D u)^{2}+a_{3}(D u)^{2} \\
\mathscr{I}_{3}^{(2)}= & b_{1}\left(D^{2} u\right)^{2}+b_{2}(D u)^{2}, \quad \hat{I}_{4}=: I_{4}:+\hbar: I_{4}^{(1)}:+\hbar^{2}: I_{4}^{(2)}:+\hbar^{3}: I_{4}^{(3)}:, \\
\mathscr{I}_{4}= & -\frac{1}{2}\left(D^{3} u\right)^{2}-7 u^{2}\left(D^{2} u\right)^{2}+\frac{7}{2}(D u)^{4}-35 u^{4}(D u)^{2}-\frac{5}{2} u^{8} \\
\mathscr{I}_{4}^{(1)}= & c_{1} u^{4}(D u)^{2}+c_{2} u^{2}\left(D^{2} u\right)^{2}+c_{3}(D u)^{4}+c_{4} u^{2}(D u)^{2} \\
& +c_{5}\left(D^{3} u\right)^{2}+c_{6}\left(D^{2} u\right)^{2}+c_{7}(D u)^{2} \\
\mathscr{I}_{4}^{(2)}= & d_{1} u^{2}\left(D^{2} u\right)^{2}+d_{2}(D u)^{4}+d_{3} u^{2}(D u)^{2} \\
& +d_{4}\left(D^{3} u\right)^{2}+d_{5}\left(D^{2} u\right)^{2}+d_{6}(D u)^{2} \\
\mathscr{I}_{4}^{(3)}= & e_{1}\left(D^{3} u\right)^{2}+e_{2}\left(D^{2} u\right)^{2}+e_{3}(D u)^{2}, \ldots,
\end{aligned}
$$

Here the coefficients $a_{1}, a_{2}, \ldots, e_{3}, \ldots$, are to be determined. A few remarks are in order. The individual terms in $\mathscr{I}_{n}^{(k)}$ are linearly independent, namely no linear combination of them will lead to a total $(\sigma)$ derivative. The choice of the set of monomials is not unique due to the freedom of addition of total $(\sigma)$ derivative terms. However, here we have chosen them to be irreducible [7], i.e., no term has its highest derivative factor occurring linearly. From the general requirement, Eqs. (3.5) and (3.8), $\hat{I}_{2}$ can have a term proportional to $\hbar$, i.e., $\mathscr{I}_{2}^{(1)}=\lambda(D u)^{2}$, with an indefinite coefficient $\lambda$. However, the effect of this new term can always be absorbed to the original form of $\mathscr{I}_{2}$ by a suitable rescaling of $\hbar$ and of the field operator $u$. Therefore $\mathscr{I}_{2}^{(1)}$ is disregarded.

Since $\hat{I}_{1}$ commute with all of : $I_{n}^{(k)}:$ it does not give any information on the form of $I_{n}^{(k)}$. Vanishing commutator of $\hat{I}_{n}$ and $\hat{I}_{2}$, on the other hand, gives severe restriction on $I_{n}^{(k)}(n \geqq 3)$ and all the undetermined coefficients are fixed uniquely by this condition. Let us write down the condition $\left[\hat{I}_{2}, \hat{I}_{n}\right]=0$ more explicitly, namely we expand it in powers of $\hbar$ and require that each coefficient should vanish. In the notation introduced in Eqs. (3.18) and (3.19) they read

$$
\begin{array}{cc} 
& {\left[\hat{I}_{2}, \hat{I}_{n}\right]=0} \\
& i:\left\{I_{2}, I_{n}\right\}:=0 \\
\hbar^{1}, & i:\left\{I_{2}, I_{n}^{(1)}\right\}:+\left[: I_{2}:,: I_{n}:\right]_{(2)}=0 \\
\hbar^{2}, & i:\left\{I_{2}, I_{n}^{(2)}\right\}:+\left[: I_{2}:,: I_{n}^{(1)}:\right]_{(2)}+\left[: I_{2}:,: I_{n}:\right]_{(3)}=0 \\
\hbar^{3}, & i:\left\{I_{2}, I_{n}^{(k)}\right\}:+\left[: I_{2}:,: I_{n}^{(k-1)}:\right]_{(2)}+\left[: I_{2}:,: I_{n}^{(k-2)}:\right]_{(3)}=0 \\
\hbar^{k+1}, & {\left[: I_{2}:,: I_{n}^{(n-1)}:\right]_{(2)}+\left[: I_{2}:,: I_{n}^{(n-2)}:\right]_{(3)}=0} \\
\ldots & {\left[: I_{2}:,: I_{n}^{(n-1)}:\right]_{(3)}=0}
\end{array}
$$

The first condition (4.5.1) is automatically satisfied, since it is just the classical result, Eq. (2.9). The last two equations $(4.5 . n+1)$ and $(4.5 . n+2)$ are satisfied 
trivially due to Eq. (3.20). (It should be remarked that $I_{2}$, having maximal order 4, has at most triple contraction.) The remaining conditions, (4.5.2) to (4.5.n) determine $I_{n}^{(1)}$ to $I_{n}^{(n-1)}$ successively. By combining the Corollary to Theorem 1 with the irreducibility condition (3.8e), we arrive at

Theorem 2. The condition of vanishing commutator with $\hat{I}_{2}$, determines the quantum operator $\hat{I}_{n}$ uniquely, if the double and triple contraction terms lie in the image of the linear operator $\mathscr{M}, E q$. (2.18). If not, such $\hat{I}_{n}$ does not exist.

By explicit calculation with the aid of a formula manipulation program [15] we have established the existence of $\hat{I}_{n}, n=3,4,5,6$, and obtained their explicit forms. The orderliness seen everywhere in the calculation together with the knowledge from other formulations $[2,3,16]$ led us to the conviction that $\hat{I}_{n}$ exists for arbitrary $n$, although an analytic proof is yet to be worked out.

Theorem 3. If $\hat{I}_{n}$ and $\hat{I}_{m}$ are operators commuting with $\hat{I}_{2}$, then they commute,

$$
\left[\hat{I}_{n}, \hat{I}_{m}\right]=0 \text {. }
$$

The proof is a quantum version of the proof [8] for the classical result $\left\{I_{n}, I_{m}\right\}=0$. We start from Jacobi's identity

$$
0=\left[\hat{I}_{2},\left[\hat{I}_{n}, \hat{I}_{m}\right]\right],
$$

in which the other two terms are zero by assumption. By expanding $\left[\hat{I}_{n}, \hat{I}_{m}\right]$ in powers of $\hbar$, we write

$$
\left[\hat{I}_{n}, \hat{I}_{m}\right]=\sum_{k=1} \hbar^{k}: J_{n, m}^{(k)}:
$$

The classical result tells that $J_{n, m}^{(1)}=0$. From the remark at the end of Sect. 3 we find that $J_{n, m}^{(k)}(k \geqq 2)$ are of even order and odd weight. By substituting Eq. (4.8) into Eq. (4.7), we get

$$
\begin{aligned}
0= & \hbar^{3} i ;\left\{I_{2}, J_{n, m}^{(2)}\right\}: \\
& +\hbar^{4}\left(i:\left\{I_{2}, J_{n, m}^{(3)}\right\}:+\left[\hat{I}_{2},: J_{n, m}^{(2)}:\right]_{(2)}\right) \\
& +\hbar^{5}\left(i:\left\{I_{2}, J_{n, m}^{(4)}\right\}:+\left[\hat{I}_{2},: J_{n, m}^{(3)}:\right]_{(2)}+\left[\hat{I}_{2},: J_{n, m}^{(2)}:\right]_{(3)}\right)+\ldots
\end{aligned}
$$

The condition that the $\hbar^{3}$-term should vanish gives $J_{n, m}^{(2)}=0$ via Theorem 1 . And similarly we get $J_{n, m}^{(3)}=J_{n, m}^{(4)}=\ldots,=0$, therefore $\left[\hat{I}_{n}, \hat{I}_{m}\right]=0$.

The numerical values for the coefficients $a_{1}, \ldots, e_{3}$ are [5]

$$
\begin{array}{ll}
a_{1}=2 / 3, & a_{2}=10, \quad a_{3}=5 / 6, \\
b_{1}=-1 / 6, & b_{2}=-5 / 6, \\
c_{1}=-140, & c_{2}=-98 / 3, \quad c_{3}=112 / 9, \quad c_{4}=-35 / 3, \\
c_{5}=-26 / 15, \quad c_{6}=-7 / 3, \quad c_{7}=7 / 30, & d_{1}=14 / 9, \quad d_{3}=35 / 3, \quad d_{4}=-4 / 45, \\
d_{1}=-91 / 3, & d_{5}=-7 / 6, \quad d_{6}=-56 / 45, \\
e_{1}=-8 / 45, & e_{2}=7 / 2, \quad e_{3}=91 / 90 .
\end{array}
$$


Determination of higher operators $I_{n}^{(k)}$ is straightforward [15]. As is clear from Eq. (4.10), the quantum operator $\hat{I}_{n}$ for arbitrary $\hbar$ is not of uniform weight in contradistinction to the classical one $I_{n}$, which is of uniform weight $2 n$. However, for a special value of $\hbar$, i.e., $\hbar=1$ (the "genuine" quantum theory?) $\hat{I}_{n}$ simplifies drastically and all the non-uniform weight terms disappear, and it is expected to lead to no particle creation as in the classical case:

$$
\begin{gathered}
\hat{I}_{2}=\frac{1}{2 \pi i} \oint \frac{d z}{z}:\left\{(D u)^{2}+u^{4}\right\} \times\left(-\frac{1}{2}\right): \\
\hat{I}_{3}(\hbar=1)=f:\left\{\left(D^{2} u\right)^{2}+15 u^{2}(D u)^{2}+u^{6}\right\}: \\
\hat{I}_{4}(\hbar=1)=\oint:\left\{\left(D^{3} u\right)^{2}+28 u^{2}\left(D^{2} u\right)^{2}-7(D u)^{4}+70 u^{4}(D u)^{2}+u^{8}\right\} \times\left(-\frac{5}{2}\right): \\
\hat{I}_{5}(\hbar=1)=\oint:\left\{\left(D^{4} u\right)^{2}+45 u^{2}\left(D^{3} u\right)^{2}-100 u\left(D^{2} u\right)^{3}-90(D u)^{2}\left(D^{2} u\right)^{2}\right. \\
\left.+210 u^{4}\left(D^{2} u\right) 2-315 u^{2}(D u)^{4}+210 u^{6}(D u)^{2}+u^{10}\right\} \times 7:, \\
\hat{I}_{6}(\hbar=1)=\oint:\left\{\left(D^{5} u\right)^{2}+66 u^{2}\left(D^{4} u\right)^{2}-660 u\left(D^{2} u\right)\left(D^{3} u\right)^{2}\right. \\
-165(D u)^{2}\left(D^{3} u\right)^{2}+495 u^{4}\left(D^{3} u\right)^{2}+220\left(D^{2} u\right)^{4}-2200 u^{3}\left(D^{2} u\right)^{3} \\
-5940 u^{2}(D u)^{2}\left(D^{2} u\right)^{2}+924 u^{6}\left(D^{2} u\right)^{2}+297(D u)^{6} \\
\left.-3465 u^{4}(D u)^{4}+495 u^{8}(D u)^{2}+u^{12}\right\} \times(-21):
\end{gathered}
$$

in which $f$ stands for

$$
\frac{1}{2 \pi i} \oint \frac{d z}{z} .
$$

Another remarkable feature of the operators $\hat{I}_{n}(\hbar=1)$ is that the lowest order term [e.g. $\left(D^{4} u\right)^{2}$ in $\left.\hat{I}_{5}\right]$ and the highest order term [e.g. $u^{10}$ in $\left.\hat{I}_{5}\right]$ have the same coefficient. Mathematical reasons for this and for the uniformity of weight as well as their physical significance should better be understood.

\section{KdV Hierarchy}

Historically it was for the $\mathrm{KdV}$ equation

$$
v_{t}=v_{\sigma \sigma \sigma}+6 v v_{\sigma},
$$

that the involutive structure of an infinite set of conserved quantities was first discovered [8]. The field $v(\sigma)$ is assumed to obey the same boundary conditions and other conditions as for $u(\sigma)$ introduced in Sect. 2. The infinite set of polynomial conserved quantities is given by

$$
K_{n}=\frac{1}{2 \pi} \int_{0}^{2 \pi} d \sigma \frac{1}{2} v(\sigma) Z_{2 n+1}(v), \quad n=1,2,3, \ldots,
$$

in which $Z_{n}(v)$ is defined recursively

$$
Z_{n+1}=\partial_{\sigma} Z_{n}+v \sum_{k=1}^{n-1} Z_{k} Z_{n-k}, \quad Z_{1}=1 .
$$


They are in involution to each other $\left\{K_{n}, K_{m}\right\}=0$ under the same Poisson bracket (2.8) as the MKdV hierarchy. By adopting $K_{2}$

$$
K_{2}=\frac{1}{2 \pi} \int_{0}^{2 \pi} d \sigma \frac{1}{2}\left(-\left(v_{\sigma}\right)^{2}+2 v^{3}\right)
$$

as a Hamiltonian we get the KdV Eq. (5.1) as a canonical equation. For the KdV hierarchy the definition of the order is the same as before but the weight, Eq. (2.5) is slightly changed,

$$
\operatorname{order}(M)=\sum n_{k}, \quad \text { weight }(M)=\sum(k+2) n_{k} .
$$

This implies that $K_{n}$ is of uniform weight $2(n+1)$ and it has both even and odd order terms.

Since the Poisson bracket for the KdV hierarchy is the same as that for the $\mathrm{MKdV}$ case, the program for finding an infinite set of quantum commuting operators goes almost parallel. We start from the ansatz

$$
\hat{K}_{n}=: K_{n}:+\sum_{k=1} \hbar^{k}: K_{n}^{(k)}:, \quad n \geqq 3,
$$

and try to determine the local counterterms $K_{n}^{(k)}$ by demanding a vanishing commutator with $\hat{K}_{2}$,

$$
\left[\hat{K}_{2}, \hat{K}_{n}\right]=0, \quad \hat{K}_{2}=: K_{2}:
$$

The conditions for the possible counterterms change slightly;

(i) max. order $\left(\mathscr{K}_{n}^{(k)}\right)=n+1-2 k$,

$$
\text { implying } \mathscr{K}_{n}^{(k)}=0 \text { for } 2 k>n-1,
$$

max. weight $\left(\mathscr{K}_{n}^{(k)}\right)=2(n+1-k)$,

$\mathscr{K}_{n}^{(k)}$ has even weight terms only.

(ii) Irreducibility.

The condition (3.8e) remain true, whereas Eq. (3.8f) is changed to

$$
\mathscr{K}_{n}^{(k)} \text { should not contain terms of the form } v^{m} \text {. }
$$

With these modifications Theorems 1-3, hold as well. Explicit calculation of commutators (which is simpler for the $\mathrm{KdV}$ case, since $\hat{K}_{2}$, has simple and double contractions only) show that the lower members of commuting quantum operators do exist. The high degree of orderliness, in this case too, convince us of the existence of the higher members as well.

The explicit forms of the lower members of the quantum commuting operators for the $\mathrm{KdV}$ hierarchy are:

$$
\begin{aligned}
\hat{K}_{3}= & \oint: \frac{1}{2}\left(\left(D^{2} v\right)^{2}-10 v(D v)^{2}+5 v^{4}\right)-\hbar \frac{5}{2}(D v)^{2}: \\
\hat{K}_{4}= & \oint: \frac{1}{2}\left(-\left(D^{3} v\right)^{2}+14 v\left(D^{2} v\right)^{2}-70 v^{2}(D v)^{2}+14 v^{5}\right) \\
& +\hbar \frac{1}{6}\left(-35\left(D^{2} v\right)^{2}+6 v(D v)^{2}\right): \\
\hat{K}_{5}= & \oint: \frac{1}{2}\left(\left(D^{4} v\right)^{2}-18 v\left(D^{3} v\right)^{2}+20\left(D^{2} v\right)^{3}+126 v^{2}\left(D^{2} v\right)^{2}\right. \\
& \left.-35(D v)^{4}-420 v^{3}(D v)^{2}+42 v^{6}\right) \\
& +\hbar \frac{1}{20}\left(193\left(D^{3} v\right)^{2}-2100 v\left(D^{2} v\right)^{2}+6300 v^{2}(D v)^{2}\right. \\
& \left.+7(D v)^{2}\right)+\hbar^{2} 21\left(D^{2} v\right)^{2}:
\end{aligned}
$$


They do not simplify at all for $\hbar=1$. This is related to the fact that the canonical dimension of the field $v[2$, as seen from Eq. (5.1) or (5.4)] is not preserved by the Poisson bracket, (2.8) and the commutation relation (3.1). This might cause some subtlety for physical applications. If one wants to preserve the canonical dimension of the field $v$, one should adopt the following Poisson bracket:

$$
\left\{v(\sigma), v\left(\sigma^{\prime}\right)\right\}_{(1)}=2 \pi\left(\partial_{\sigma}^{3} \delta\left(\sigma-\sigma^{\prime}\right)+2\left(v(\sigma)+v\left(\sigma^{\prime}\right)\right) \partial_{\sigma} \delta\left(\sigma-\sigma^{\prime}\right)\right) .
$$

This is the second member (the first member is (2.8)) of the infinite set of "Poisson brackets" for the KdV hierarchy [11],

$$
\begin{gathered}
\{P, Q\}_{(N)}=2 \pi \int_{0}^{2 \pi} d \sigma \frac{\delta P}{\delta v} M^{N} \frac{\partial}{\partial \sigma} \frac{\delta Q}{\delta v}, \quad N=0,1,2, \ldots, \\
M=\left(\partial_{\sigma}\right)^{2}+2 v(\sigma)+2 \partial_{\sigma} v(\sigma) \partial_{\sigma}^{-1},
\end{gathered}
$$

under which the set $K_{n}, n=1,2,3, \ldots$, is in involution

$$
\left\{K_{n}, K_{m}\right\}_{(N)}=0 .
$$

In fact, the Poisson bracket (5.10) has been discussed by Gervais [3] in connection with the Virasoro algebra. It is not, however, straightforward to quantize the $v$-field starting from the Poisson bracket (5.10).

\section{Summary and Comments}

By applying field theoretical techniques, we have almost established the existence of an infinite set of quantum commuting operators of the MKdV hierarchy. Our method gives an algorithm to determine the explicit forms of these operators. For the KdV hierarchy similar results are obtained. These sets of operators may constitute the Cartan subalgebra of an infinite rank Lie algebra which might underlie the quantum integrable systems of some sort.

A lot of interesting things remain to be elucidated. We remark on only a few of them here. Clear understanding of the reasons for the existence of $\hat{I}_{n}$ is vital for further steps. Is there a recursion formula linking $\hat{I}_{n}$ 's? An immediate next step for a quantum field theory would be to find a complete set of states constituting the simultaneous eigenstates of the $\hat{I}_{n}$ 's. This is also connected to understanding the relationship [16] between our naive continuum approach and the conventional quantum inverse method or the transfer matrix approach based on a lattice. The applicability of the present method to other broader types of soliton equations is certainly an interesting point.

Acknowledgements. We are grateful to Professors T. Kimura, K. Okamoto, A. Fujimoto, M. Wadati, and Dr. M. Omote for useful discussion.

\section{References}

1. See for example, Scott, A.C., Chu, F.Y.F., McLaughlin, D.W.: Proc. IEEE 61, 1443-1483 (1973)

Bullough, R.K., Caudrey, P.J.: Solitons. Bullough, R.K., Caudrey, P.J. (eds.): Berlin, Heidelberg, New York: Springer 1980 
2. Faddeev, L.D.: Les Houches lecture notes, Session XXXIX. Amsterdam: Elsevier 1984

3. Gervais, J.-L.: Infinite family of polynomial functions of the Virasoro generators with vanishing Poisson brackets, and transport matrices associated with the Virasoro algebra. Phys. Lett. 160B, 277-278, 279-282 (1985)

4. Sklyanin, E.K.: Method of the inverse scattering problem and the nonlinear quantum Schrödinger equation. Sov. Phys. Dokl. 24, 107-109 (1979)

Thacker, H.B., Wilkinson, D.: Inverse scattering transform as an operator method in quantum field theory. Phys. Rev. D 19, 3660-3665 (1979)

5. Sasaki, R., Yamanaka, I.: In Essays in Honor of the 60th Birthday of Professor Yoshio Yamaguchi. Terazawa, H., Sakai, N. (eds.). Singapore: World Scientific 1986

6. Konno, K., Sanuki, H., Ichikawa, Y.-H.: Conservation laws of nonlinear-evolution equations. Prog. Theor. Phys. 52, 886-889 (1974)

Wadati, M., Sanuki, H., Konno, K.: Relationships among inverse method, Backlund transformation and an infinite number of conservation laws. Prog. Theor. Phys. 53, 419-436 (1975)

7. Kruskal, M.D., Miura, R.M., Gardner, C.S., Zabusky, N.J.: Korteweg-de Vries equation and generalizations. V. Uniqueness and nonexistence of polynomial conservation laws. J. Math. Phys. 11, 952-960 (1970)

8. Gardner, C.S.: Korteweg-de Vries equation and generalizations. IV. The Korteweg-de Vries equation as a Hamiltonian system. J. Math. Phys. 12, 1548-1551 (1971)

9. Flaschka, H., Newell, A.C.: In Lecture Notes in Physics, Vol. 38. Moser, J. (ed.). Berlin, Heidelberg, New York: Springer 1975

Newell, A.C.: The general structure of integrable evolution equations. Proc. R. Soc. A 365, 283-311 (1979)

10. Kodama, Y., Wadati, M.: Theory of canonical transformations for nonlinear evolution equations. Prog. Theor. Phys. 56, 342-343; 1740-1755 (1976)

11. Alberty, J., Koikawa, T., Sasaki, R.: Canonical structure of soliton equations. I. Physica 5D, 43-65 (1982)

Sasaki, R.: Geometric approach to soliton equations. Proc. R. Soc. A 373, 373-384 (1980)

12. Hietarinta, J.: Classical versus quantum integrability. J. Math. Phys. 25, 1833-1840 (1984)

Calogero, F., Ragnisco, O., Marchioro, C.: Exact solution of the classical and quantal onedimensional many-body problems with the two-body potential $V_{a}(x)=g^{2} a^{2} / \sinh ^{2}(a x)$. Lett. Nuovo Cimento 13, 383-387 (1975)

Perelomov, A.M.: Exact results for one-dimensional many-particle systems. Sov. J. Part. Nucl. 10, 336-348 (1979)

13. Fubini, S., Hanson, J., Jackiw, R.: New approach to field theory. Phys. Rev. D 7, 1732-1760 (1973)

14. See for example, Bjorken, J., Drell, S.: Relativistic quantum fields. New York: McGraw-Hill 1965

15. Yamanaka, I.: Hiroshima Preprint RRK 86 (in preparation)

16. Omote, M., Sasaki, R., Yamanaka, I.: Hiroshima Preprint RRK 86-46

Communicated by $\mathrm{H}$. Araki

Received August 11, 1986 